\title{
Zum Bilingualismus im tschechischen Bildungssystem \\ Tschechisch-deutsche Bildungsgänge an tschechischen Schulen und die Charakteristik der SchülerInnen
}

\section{Martin Lachout}

The article deals with Czech-German bilingualism, as it is practiced today in the Czech Republic. First, the author defines the terms "multilingualism" - while discussing the distinction between the German "Vielsprachigkeit" and "Mehrsprachigkeit" - and "bilingualism", delimits this phenomenon within a psycholinguistic approach, and analyzes it from the sociolinguistic point of view. After having provided the theoretical background for an analysis, the author introduces his pilot study on multi- and bilingualism. There, he establishes a working model accounting for fluency in both Czech and German languages, as it is promoted and practiced in bilingual preschools, elementary, middle, and high schools in the Czech Republic. The article concludes with observations on this particular type of a bilingual environment, with special attention to its functioning with respect to the ratio of simultaneously, or successively bilingual children/students to those with a monolingual background.

Bilingualism - German in the Czech Republic - multi-lingual education

Der vorliegende Beitrag widmet sich der Problematik des tschechisch-deutschen Bilingualismus in der Tschechischen Republik von heute. In der Einleitung definiert der Autor zunächst die Termini „Vielsprachigkeit“, „Mehrsprachigkeit“ und „Bilingualismus“, anschließend grenzt er dieses Phänomen aus Sicht der Psycholinguistik ab, um im zweiten Schritt ganz kurz die soziolinguistische Perspektive zu thematisieren. Im Anschluss an die theoretischen Einführung präsentiert der Autor seine Pilotstudie, in deren Rahmen er sich vor allem auf diejenigen Institutionen konzentriert hat, die die beschriebene Art des tschechischdeutschen Bilingualismus mit ihren bilingualen Bildungsgängen unterstützen, d. h. auf zweisprachige Kindergärten, Haupt- und Mittelschulen in der Tschechischen Republik. Die in diesen Bildungsinstitutionen geförderte Mehrsprachigkeit wird dann im Hinblick auf das jeweilige Verhältnis der sog. simultan und sukzessiv bilingualen Kinder/Schüler zu den monolingualen untersucht.

Bilingualismus - Deutsch in Tschechien - mehrsprachige Erziehung

Je mehr Sprachen du sprichst, desto mehr bist du Mensch. (Tschechisches Sprichwort)

\section{Einführung: Die Zielsetzung des Aufsatzes}

Im theoretischen Teil dieses Aufsatzes haben wir uns zum Ziel gesetzt, das Phänomen des Bilingualismus allgemein und v. a. aus Sicht der Sprachpsychologie (heute eher der Psycholinguistik) zu beschreiben und zu klassifizieren. Kurz widmen wir unsere Aufmerksamkeit auch den Vorteilen des Bilingualismus. 
Im zweiten Teil war unser Anliegen, sich eine Übersicht über solche Institutionen zu verschaffen, die den tschechisch-deutschen Bilingualismus unterstützen und fördern, d. h. über solche Institutionen, die ihren Schülern bilinguale Bildungsgänge anbieten und auch nach den entsprechenden Prinzipien unterrichten. Es handelte sich um Bildungsinstitutionen der präprimären, primären und sekundären Ausbildungsstufe; konkret haben wir Kindergärten, Hauptschulen und Gymnasien in ganz Tschechien angesprochen. Neben der o. g. Übersicht setzen wir uns zum Ziel, die Leser mit den Zahlen der tschechisch-deutsch zweisprachigen Schüler bekannt zu machen und die Schüler bestimmten Typen von Bilingualismus zuzuordnen. Wir gehen dabei von folgenden Annahmen aus:

1. Über ausführliche Daten zu den in der Tschechischen Republik laufenden bilingualen Bildungsgängen verfügt das Ministerium für Schulwesen, Jugend und Sport, das genaue Daten zu diesen Bildungsgängen registriert.

2. Die meisten Institutionen werden von ausländischen Subjekten finanziert; die Unterstützung und Weiterförderung der bilingualen tschechisch-deutschen Bildungsgänge (und damit zugleich der deutschen Sprache) erfolgt also nicht durch den tschechischen Staat, sondern durch ausländische Träger.

3. Schüler, die sog. simultan tschechisch-deutsch zweisprachig sind, d. h. aus gemischten Familien stammen, sollten in diesen Institutionen stark vertretet sein.

\section{Zum Wesen des Bilingualismus}

Im heutigen Europa ist die Kenntnis mehrerer Sprachen unumstritten ein Muss. Es reicht lange nicht mehr nur die Muttersprache zu sprechen; es ist nicht einmal ausreichend, neben der Muttersprache Englisch zu sprechen, denn das Beherrschen des Englischen stellt heute sozusagen die Grundkompetenz jedes EU-Bürgers dar. Eine wichtige Rolle spielt darum im Europa von heute die Mehrsprachigkeit, zusammen mit der Vielsprachigkeit. Beide Termini sind allerdings voneinander zu unterscheiden. Während sich die Mehrsprachigkeit auf die Kenntnis zweier oder mehrerer Sprachen bezieht, die ein Mensch im Rahmen einer Gesellschaft spricht, bedeutet die Vielsprachigkeit die parallele Existenz verschiedener Sprachen in einer bestimmten Gesellschaft (Europarat 2001: 17).

Auf Grund des Gesagten unterscheiden wir den sog. individuellen Bilingualismus, der sich auf die sprachliche Kompetenz eines Individuums bezieht (wobei die gesellschaftlichen Umstände nicht berücksichtigt werden), vom kollektiven Bilingualismus, der ein gesellschaftliches Phänomen darstellt, bei dem die Bevölkerung eines Landes (einer Region) in ständiger Interaktion mit mehreren Sprachen steht.

An Bedeutung hat in den letzten Jahren jedoch vor allem das Phänomen der Mehrsprachigkeit im Sinne des individuellen Bilingualismus gewonnen. 
Der häufigste Typ der Mehrsprachigkeit ist wohl die Zweisprachigkeit, auch Bilingualismus genannt. Laut Apeltauer (1987) ist „Zweitspracherwerb [...] jede Aneignung einer weiteren Sprache (neben der Muttersprache, wobei die einzelnen Formen dieses Aneignungsprozesses sich nach Lernalter (gleichzeitig/nachzeitig zum Erstspracheerwerb) und Lernkontext (natürlich/gesteuert) weiter differenzieren lassen." Unter dem Begriff Bilingualismus verstehen wir demnach die Eigenschaft eines Menschen alternativ zwei Sprachcodes zu benutzen, ohne Rücksicht auf die aktuelle kommunikative Situation.

Eine der ersten Definitionen des Begriffs finden wir allerdings schon in der ersten Hälfte des 20. Jahrhunderts bei dem amerikanischen Linguisten Bloomfield, nach dessen Ansicht „Bilingualismus eine Fähigkeit ist, zwei Sprachen auf dem Niveau eines Muttersprachlers zu beherrschen [...]. Man kann selbstverständlich nicht die Stufe der Vollkommenheit definieren, auf der aus einem Nichtmuttersprachler ein Mehrsprachiger wird: Die Unterscheidung ist relativ" (Bloomfield 1933, zit. nach Harding-Esch und Riley 2008: 40). ${ }^{1}$

Diese Definition scheint uns jedoch ein wenig zu radikal zu sein. Selbst die oben genannten Autoren Harding-Esch und Riley (2008) machen auf die Tatsache aufmerksam, dass kein Mensch imstande ist, eine Sprache in ihrer Vollkommenheit zu erlernen. Dies betrifft paradoxerweise auch unsere eigene Muttersprache. Damit widerlegen Harding-Esch und Riley die Definition von Bloomfield, in der die vollständige Sprachkompetenz als das perfekte Beherrschen einer Sprache verstanden wird. „Niemand wendet seine eigene Sprache in ihrem vollen Umfang an [...]. Jeder von uns benutzt nur einen Teil von seiner Muttersprache" (Harding-Esch und Riley 2008: 39). So enthält der Wortschatz einer Sprache mehrere Hunderttausend Lexeme und Phraseme, die kein einzelner Sprecher in ihrer Gesamtheit kennt. Überdies verfügt jede Sprache über spezielle Bereiche (Fachtermini, Fachsprache, Jargonismen, Professionalismen usw.), die nur Personen aus dem jeweiligen gesellschaftlichen Bereich zugänglich sind.

Einer der wohl bekanntesten Versuche, eine allgemeingültige Definition für Bilingualismus zu geben, findet man bei Skutnabb-Kangas, die Bilingualismus folgendermaßen definiert: ${ }^{2}$

A bilingual speaker is someone who is able to function in two (or more) languages, either in monolingual or bilingual communities, in accordance with the sociocultural demands made by an individual's communicative and cognitive competence by these communities or by the individual herself, at the same level as native speakers, and who is able positively to identify with both (or all) language groups (and cultures) or parts of them. (SkutnabbKangas 1984: 90)

1 Übersetzt vom Autor des vorliegenden Artikels.

2 Es sei dabei an dieser Stelle darauf hingewiesen, dass sich diese Definition eher auf ein bilinguales Individuum bezieht, da hier auch der kulturelle Aspekt betont wird. 
Dagegen ist für John McNamara (1967: 59-60) jeder zweisprachig, der neben seiner Muttersprache in einer anderen Sprache sprechen, verstehen, schreiben oder lesen kann. Richtet man sich dagegen nach Bloomfield, wird man kaum jemand als zweisprachig bezeichnen, der eine Fremdsprache nur rezeptiv beherrscht. Von einem „muttersprachlichem Niveau“ kann dann ja nicht die Rede sein. Eine m. E. vernünftige Mittelposition nehmen in ihrer Definition von „Bilingualität“ Jonekeit und Kielhöfer (2002) ein. Sie sprechen von dem Bewusstsein der Zweisprachigkeit, wobei es sich um das subjektive Gefühl handelt, sich in beiden Sprachen wohl zu fühlen. Nach Jonekeit und Kielhöfer kann die objektive Beurteilung darüber, ob eine Person die sprachliche Fertigkeiten und Fähigkeiten perfekt beherrscht, sehr unterschiedlich ausfallen.

Wenn wir von „Bilingualismus“ sprechen, können wir dieses Phänomen aus zwei Blickwinkeln betrachten: zum einen aus dem Blickwinkel der Psycholinguistik, zum anderen aus dem Blickwinkel der Soziologie.

Aus Sicht der Psycholinguistik sprechen wir von Bilingualismus, wenn ein Mensch zwei bzw. mehrere Sprachcodes (Sprachen) in vollem Umfang beherrscht (also auf dem Niveau der Muttersprache) und fähig ist diese wechselweise einzusetzen. Ist der Sprecher im Stande auch mehrere Sprachcodes zu benutzen, wird manchmal von "Multilinguismus" gesprochen. So verstehen etwa Průcha et al (2003: 25) unter dem Begriff Bilingualismus „die Fähigkeit eines Individuums zwei Sprachen sprechen zu können", wobei es sich aus Sicht der Psycholinguistik um eine gewisse „Art von kommunikativer Kompetenz handelt, die es ermöglicht, verschiedene Kommunikationsbedürfnisse sowohl mit Hilfe der Erst- als auch der Zweitsprache zu realisieren".

Das Phänomen des Bilingualismus lässt sich aus Sicht der Psycholinguistik auf Grund von verschiedenen Gesichtspunkten klassifizieren. Das größte Problem dabei ist, diejenigen Kriterien zu bestimmen, die das Wesen des Bilingualismus am besten erfassen. Laut Skutnabb-Kangas (1984) zählen dazu folgende Kriterien:

- Herkunftskriterium (von wem, wann und wie wir beide Sprachen (er)lernen);

- Kompetenzkriterium (Niveau der Beherrschung beider Sprachen);

- Anwendungskriterium (wie wir beide Sprachen anwenden);

- Kriterium der Identifizierung mit der Sprache (inwieweit identifiziert sich der Sprecher mi den Sprachen und inwieweit wird er als Sprecher der konkreten Sprache von anderen Sprechern akzeptiert?).

Um einen Gesamteindruck zu vermitteln, führen wir an dieser Stelle eine überschaubare Aufzählung der möglichen Klassifizierungsmerkmale an, wie sie z. B. Jelínek (2004-2005: 53-155 und 2005-2006: 8-11) und Muhić (2004: 65 ff.) nennen.

Aus der Sicht der Zeitspanne, in der ein Mensch die beiden Sprachcodes erlernt hat, unterscheiden wir: 
a) den simultanen Bilingualismus - paralleler Erwerb beider Codes von Geburt an,

b) den sukzessiven Bilingualismus, manchmal als sequenzieller Bilingualismus bezeichnet - ein Mensch erwirbt den zweiten Sprachcode erst nach dem Erwerb des ersten Codes.

Nach dem Maß der Kompetenz, die der Sprecher in beiden Sprachen erreicht, unterscheiden wir:

a) den symmetrischen Bilingualismus - der Sprecher beherrscht beide Sprachen auf gleichem Niveau,

b) den asymmetrischen Bilingualismus - eine Sprache (gewöhnlich die erste) ist dominant im Vergleich zur zweiten Sprache.

Nach dem Alter, in dem beide Sprachen erworben werden, lässt sich unterscheiden:

a) der infantile Bilingualismus - beide Sprachen werden von Geburt an erworben,

b) der adoleszente Bilingualismus - die eine Sprache wird erst in höherem Alter erworben (oft handelt es sich hier um eine Aneignung durch bewusstes Lernen),

c) derspäte Bilingualismus-Erwerb/Erlernen einer Sprache erst im Erwachsenenalter. Nach dem Umfeld, in dem der Sprecher die Sprachen erworben hat, sind zu unterscheiden:

a) der primäre oder auch natürliche Bilingualismus - im Rahmen der Familie, in der Regel von Geburt an,

b) der sekundäre oder auch künstliche Bilingualismus - gewöhnlich mit der institutionellen Ausbildung verbunden.

\section{Kurzer Exkurs zum Bilingualismus aus soziolinguistischer Sicht}

Aus Sicht der Soziolinguistik verstehen wir unter dem Begriff Bilingualismus den Gebrauch zweier Sprachcodes (siehe oben - kollektiver Bilingualismus), wobei das Ausmaß ihrer Beherrschung keine relevante Rolle spielt. Soziolinguistisch gesehen reicht es nämlich, „dass im Rahmen einer Gesellschaft nebeneinander zwei Sprachen existieren, ohne Rücksicht darauf, wie viele Menschen die Sprache sprechen oder in wie weit sie sie beherrschen" (Černý 1996: 397).

Diese Art des Bilingualismus wird dann als kollektiver Bilingualismus bezeichnet. In diesem Fall können wir oft einen pragmatisch gesteuerten Gebrauch beider Sprachcodes beobachten: Den einen Sprachcode setzen die Sprecher z. B. im Privatleben ein, den anderen beispielsweise in der Schule, im Verkehr mit Behörden usw. Typische Beispiele für Länder mit kollektivem Bilingualismus sind z. B. die Schweiz, Kanada oder Belgien. Bis 1993 war auch die ehemalige Tschechoslowakei ein solches Beispiel. 


\section{Vorteile des Bilingualismus für das Individuum}

Im Unterschied zu den früheren Annahmen, dass die Mehrsprachigkeit für einen Menschen schädlich sein könnte, dass sie ihn in seiner Entwicklung (dies gilt vor allem für Kinder) inhibieren oder es zu Sprachstörungen kommen kann, wissen wir heute, dass der Bilingualismus gerade umgekehrte Folgen hat. Laut Štefánik (2000: 31) hat Bilingualismus für den jeweiligen Sprecher zahlreiche Vorteile:

- Das Beherrschen von zwei Sprachen ermöglicht einen besseren Zugang zu einem größeren Informationspensum.

- Ein bilinguales Kind kann für den Erwerb neuer Kenntnisse beide Sprachen nutzen.

- Mit Hilfe der anderen Sprache kann das Kind besser die Kultur der Sprachregion kennenlernen, die durch Bücher, Lieder, Sprichwörter, Redewendungen $u$. a. vermittelt wird.

- Ein mehrsprachiges Kind hat in der Zukunft bessere Chancen beim Studium oder bei der Arbeitssuche; mittlerweile ist die Fähigkeit, gleichzeitig in mehreren Sprachen kommunizieren zu können, eine häufige und wichtige Forderung vieler Arbeitgeber.

- Die Fähigkeit, sich in zwei oder auch mehreren Sprachen zu verständigen, zeigt sich positiv beim Reisen oder beim Anknüpfen neuer Kontakte; die Kenntnis nur einer einzigen Sprache stellt oft eine nicht überwindbare Barriere beim Aufbau einer engeren Beziehung dar, weshalb ein Mehrsprachiger oft mehr Freunde hat als ein Einsprachiger.

- In gemischten Ehen, wo ein Elternteil die Muttersprache seines Partners gar nicht oder nur auf elementarem Niveau beherrscht, besitzt das Kind die Fähigkeit mit beiden Elternteilen in ihrer Muttersprache zu kommunizieren; dank dessen kann das Kind unmittelbar auch mit Großeltern, Tanten, Onkeln und anderen Verwandten beider Eltern kommunizieren.

- Bilinguale Kinder sind sich dessen bewusst, dass die Sprache ein Kommunikationsmittel ist; sie sind im Stande mit der Sprache zu „spielen“ und dadurch ihr Denken zu entfalten und ihre Kreativität zu verbessern.

Zusammenfassend lässt sich sagen, dass sich das Beherrschen von zwei oder auch mehreren Sprachen positiv auf alle kognitiven Vorgänge auswirkt und dass dadurch insbesondere das abstrakte Denken gefördert wird. Außerdem leiden zweisprachige Sprecher weniger unter Vorurteilen.

An dieser Stelle wäre noch zu ergänzen, dass zweisprachig aufwachsende Kinder in ihrer kognitiven Entwicklung davon profitieren, dass sie ständig - sei es bewusst oder unbewusst - gezwungen sind, zwei unterschiedliche Sprachsysteme zu vergleichen. 
Der nächste Punkt, in dem mehrsprachige Kinder den einsprachigen überlegen sind, zeigt sich bei der Kompensation sprachlicher Defizite (vgl. z. B. Lachout 2014). Hier weisen solche Kinder einen deutlichen kognitiven Vorsprung gegenüber einsprachigen Kindern auf. Auch bei der Aufmerksamkeitskontrolle haben mehrsprachige Kinder einen deutlichen Vorsprung, wenn es darum geht, verschiedene Störfaktoren bei der Kommunikation zu vermeiden (vgl. Bialystok 2001).

\section{Zur Pilotstudie}

Das Ziel unserer Pilotstudie war es u. a., diejenigen Bildungsinstitutionen zu ermitteln, in denen zweisprachige tschechisch-deutsche Bildungsgänge angeboten werden. Um relevante Informationen zu gewinnen, haben wir uns an das für unsere Frage zuständige Dezernat des Tschechischen Ministeriums für Schulwesen, Jugend und Sport gewandt. Unsere Erwartungen waren, dass wir gerade von diesem Amt alle Informationen über bilinguale Schulen, ihre Ausbildungsprogramme bzw. Finanzierungsart erhalten würden, von welchen wir weiter bei unserer Arbeit ausgehen könnten. Zu unserer Überraschung haben wir festgestellt, dass hier keine für uns relevanten Angaben zu erhalten waren. Uns wurden lediglich Namen von einigen Schulen mitgeteilt, wobei es sich bei Weitem nicht um alle relevanten Schulen handelte. Deshalb mussten wir einen anderen Weg gehen, und zwar im Internet recherchieren um geeignete Schulen direkt ansprechen zu können.

Mit Hilfe einer Internet-Recherche ist es uns letztendlich gelungen, die von uns gewünschten Bildungsinstitutionen zu finden. Wir sind uns jedoch dessen bewusst, dass es sich um Informationen ohne Gewähr auf Vollständigkeit handelt.

Wie schon erwähnt, ging es uns um Objekte von institutionellem Vorschul-, Hauptschul- und Mittelschulcharakter. Noch vor unserer eigentlichen Forschung haben wir versucht, den aktuellen Stand der Schülerzahlen mit nicht-tschechischer Nationalität (im Idealfall mit deutscher, österreichischer oder schweizerischer) und die Zahl von Kindern und Jugendlichen, die Deutsch als Fremdsprache lernen, ins Visier zu nehmen. Auch in diesem Punkt waren wir beim Schulministerium nicht erfolgreich. Auch hier erhielten wir nur allgemeine, zusammenfassende Statistiken, mit denen wir im Folgenden arbeiten. Detaillierte statistische Angaben, die die Schülerzahlen konkret nach ihrer Nationalität aufschlüsseln würden, waren vom Ministerium nicht in Erfahrung zu bringen.

\subsection{Schüler in bilingualen Bildungsgängen}

Laut Angaben im Statistischen Jahrbuch für das Jahr 2014 (zum Herunterladen auf den Web-Seiten des Tschechischen Amtes für Statistik) hatten im Schuljahr 2013/2014 in der präprimären Ausbildungsstufe 650 Kinder, im primären 1413 
Schüler/Schülerinnen und auf der Ebene der Mittelschulen noch 560 Schüler/ Schülerinnen die Staatsangehörigkeit eines Mitgliedslandes der EU (außer Tschechien und der Slowakei). Diese Statistiken geben jedoch keinen eindeutigen Aufschluss darüber, um welche Nationalitäten es sich genau handelt, deshalb konnten wir auf Grund der Tabellen überhaupt nicht erschließen, wie viele Kinder der deutschen/österreichischen Nationalität zuzurechnen waren. Auf unsere beim Ministerium gezielt gestellte Frage haben wir wiederum keine befriedigende Antwort erhalten. Laut der Äußerung des Ministeriums werden solch detaillierte Daten nicht erhoben. Leider konnten wir anhand der Angaben keine für unsere Studie relevanten Zahlen ableiten. Die unten angeführten Tabellen dienen daher nur dem Zweck, eine allgemeine Übersicht zu geben.

\begin{tabular}{|c|c|c|c|c|c|c|c|}
\hline Ausbildung & & & & & & & EDUCATION \\
\hline \multicolumn{8}{|c|}{ Kinder in Kindergärten nach Staatsangehörigkeit Children attending nursery schools by citizenship } \\
\hline \multirow{2}{*}{ Indikator } & \multicolumn{6}{|c|}{ Schuljahr/ School year } & \multirow{2}{*}{ Indicator } \\
\hline & $2005 / 06$ & $2009 / 10$ & $2010 / 11$ & $2011 / 12$ & $2012 / 13$ & $2013 / 14$ & \\
\hline Kinder insgesamt & 282183 & 314008 & 328612 & 342521 & 354340 & 363568 & Children, total \\
\hline \multicolumn{8}{|l|}{ darunter } \\
\hline $\begin{array}{l}\text { Tschechische } \\
\text { Republik }\end{array}$ & 278970 & 310045 & 324389 & 337807 & 348906 & 357261 & Czech Republic \\
\hline Slowakei & 435 & 639 & 648 & 786 & 886 & 1096 & Slovakia \\
\hline $\begin{array}{l}\text { andere EU- } \\
\text { Länder }\end{array}$ & 238 & 401 & 409 & 403 & 505 & 650 & \begin{tabular}{|l|} 
Other EU \\
countries
\end{tabular} \\
\hline $\begin{array}{l}\text { andere europ. } \\
\text { Länder }\end{array}$ & 997 & 1389 & 1585 & 1717 & 1929 & 2231 & $\begin{array}{l}\text { Other } \\
\text { European } \\
\text { countries }\end{array}$ \\
\hline $\begin{array}{l}\text { andere Länder } \\
\text { der Welt }\end{array}$ & 1543 & 1534 & 1581 & 1808 & 2114 & 2330 & $\begin{array}{l}\text { Other countries } \\
\text { of the world }\end{array}$ \\
\hline
\end{tabular}

Tabelle Nr. 1: Kinder in Kindergärten nach Staatsangehörigkeit (Quelle: Statistická ročenka České republiky 2014, Čsú) ${ }^{1}$

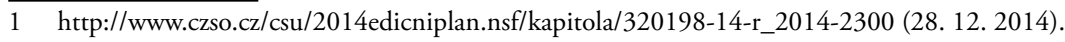




\begin{tabular}{|c|c|c|c|c|c|c|c|}
\hline \multicolumn{2}{|l|}{ Ausbildung } & & & & & & EDUCATION \\
\hline \multicolumn{8}{|c|}{ Schüler in Grundschulen nach Staatsangehörigkeit } \\
\hline \multicolumn{8}{|c|}{ Pupils of basic schools by citizenship } \\
\hline \multirow{3}{*}{ Indikator } & \multicolumn{6}{|c|}{ Schuljahr } & \multirow{3}{*}{ Indicator } \\
\hline & & & Schoo & year & & & \\
\hline & $2005 / 06$ & $2009 / 10$ & $2010 / 11$ & $2011 / 12$ & $2012 / 13$ & $2013 / 14$ & \\
\hline \begin{tabular}{|l|}
$\begin{array}{l}\text { Schüler } \\
\text { insgesamt }\end{array}$ \\
\end{tabular} & 916575 & 794459 & 789486 & 794642 & 807950 & 827654 & Pupils, total \\
\hline \multicolumn{8}{|l|}{ darunter } \\
\hline \begin{tabular}{|l|} 
Tschechische \\
Rep. \\
\end{tabular} & 904296 & 780620 & 775377 & 780298 & 793399 & 812545 & Czech Republic \\
\hline Slowakei & 2074 & 2805 & 2905 & 3161 & 3285 & 3439 & Slovakia \\
\hline \begin{tabular}{|l} 
andere EU- \\
Länder \\
\end{tabular} & 542 & 976 & 1009 & 1076 & 1186 & 1413 & \begin{tabular}{|l|}
$\begin{array}{l}\text { Other EU } \\
\text { countries }\end{array}$ \\
\end{tabular} \\
\hline \begin{tabular}{|l|} 
andere europ. \\
Länder
\end{tabular} & 4762 & 5233 & 5418 & 5565 & 5627 & 5702 & $\begin{array}{l}\text { Other European } \\
\text { countries }\end{array}$ \\
\hline $\begin{array}{l}\text { andere Länder } \\
\text { der Welt }\end{array}$ & 4901 & 4825 & 4777 & 4542 & 4453 & 4555 & $\begin{array}{l}\text { Other countries } \\
\text { of the world }\end{array}$ \\
\hline
\end{tabular}

Tabelle Nr. 2: SchülerInnen in Grundschulen nach Staatsangehörigkeit. (Quelle: Statistická ročenka České republiky 2014, ČSÚ) ${ }^{1}$

\begin{tabular}{|c|c|c|c|c|c|c|c|}
\hline Ausbildung & & & & & & & EDUCATION \\
\hline \multicolumn{8}{|c|}{ Schüler in Mittelschulen nach Staatsangehörigkeit } \\
\hline \multicolumn{8}{|c|}{ Pupils at secondary schools by citizenship } \\
\hline \multirow{3}{*}{ Indikator } & \multicolumn{6}{|c|}{ Schuljahr } & \multirow{3}{*}{ Indicator } \\
\hline & & & Schoo & year & & & \\
\hline & $2005 / 06$ & $2009 / 10$ & $2010 / 11$ & $2011 / 12$ & $2012 / 13$ & $2013 / 14$ & \\
\hline $\begin{array}{l}\text { Schüler } \\
\text { insgesamt }\end{array}$ & 577605 & 556260 & 532889 & 501185 & 470725 & 448745 & Pupils, total \\
\hline \multicolumn{8}{|l|}{ darunter } \\
\hline $\begin{array}{l}\text { Tschechische } \\
\text { Rep. }\end{array}$ & 572665 & 548360 & 524431 & 492333 & 461701 & 439598 & Czech Republic \\
\hline Slowakei & 739 & 1373 & 1540 & 1530 & 1574 & 1652 & Slovakia \\
\hline $\begin{array}{l}\text { andere EU- } \\
\text { Länder }\end{array}$ & 360 & 541 & 521 & 474 & 467 & 560 & $\begin{array}{l}\text { Other EU } \\
\text { countries }\end{array}$ \\
\hline $\begin{array}{l}\text { andere europ. } \\
\text { Länder }\end{array}$ & 2244 & 3029 & 3276 & 3616 & 3754 & 3738 & $\begin{array}{l}\begin{array}{l}\text { Other European } \\
\text { countries }\end{array} \\
\end{array}$ \\
\hline $\begin{array}{l}\text { andere Länder } \\
\text { der Welt }\end{array}$ & 1597 & 2957 & 3121 & 3232 & 3229 & 3197 & $\begin{array}{l}\text { Other countries } \\
\text { of the world }\end{array}$ \\
\hline & & & & & \multicolumn{3}{|c|}{$\begin{array}{l}\text { 1) A pupil studying at more schools } \\
\text { at the same time is counted more } \\
\text { times; a pupil studying more fields of } \\
\text { education at one school is counted } \\
\text { only ones at that school. }\end{array}$} \\
\hline
\end{tabular}

Tabelle Nr. 3: SchülerInnen in Mittelschulen nach Staatsangehörigkeit. (Quelle: Statistická ročenka České republiky 2014, ČSú)2

1 http://www.czso.cz/csu/2014edicniplan.nsf/kapitola/320198-14-r_2014-2300 (28. 12. 2014).

2 http://www.czso.cz/csu/2014edicniplan.nsf/kapitola/320198-14-r_2014-2300 (28. 12. 2014). 
Nicht einmal die Informationen, die in Tabelle 4 angeführt sind, waren für unsere Studie von besonderem Nutzen.

\begin{tabular}{|c|c|c|c|c|c|c|c|}
\hline AUSBILDUNG & & & & & & & EDUCATION \\
\hline \multicolumn{8}{|c|}{ Grundschulen nach der unterrichteten Sprache } \\
\hline \multicolumn{8}{|c|}{ Basic schools by teaching language } \\
\hline \multirow{3}{*}{ Indikator } & \multicolumn{6}{|c|}{ Schuljahr } & \multirow{3}{*}{ Indicator } \\
\hline & \multicolumn{6}{|c|}{ School year } & \\
\hline & $2005 / 06$ & $2009 / 10$ & $2010 / 11$ & $2011 / 12$ & $2012 / 13$ & $2013 / 14$ & \\
\hline $\begin{array}{l}\text { Schulen } \\
\text { insgesamt }\end{array}$ & 4474 & 4125 & 4123 & 4111 & 4095 & 4095 & Schools, total \\
\hline $\begin{array}{l}\text { darunter mit } \\
\text { der Sprache: }\end{array}$ & & & & & & & $\begin{array}{l}\begin{array}{l}\text { Teaching } \\
\text { language }\end{array} \\
\end{array}$ \\
\hline Tschechisch & 4450 & 4100 & 4101 & 4089 & 4073 & 4074 & Czech \\
\hline $\begin{array}{l}\text { Tschechisch } \\
\text { u. Deutsch } \\
\text { (bilingual) }\end{array}$ & 1 & 1 & 1 & 1 & 1 & - & $\begin{array}{l}\text { Czech and } \\
\text { German } \\
\text { (bilingual) }\end{array}$ \\
\hline $\begin{array}{l}\text { Tschechisch } \\
\text { u. Englisch } \\
\text { (bilingual) }\end{array}$ & & 3 & - & - & - & - & $\begin{array}{l}\text { Czech and } \\
\text { English } \\
\text { (bilingual) }\end{array}$ \\
\hline Polnisch & 23 & 21 & 21 & 21 & 21 & 21 & Polish \\
\hline Klassen insgesamt & 45769 & 41941 & 41720 & 42105 & 41739 & 42334 & Classes, total \\
\hline $\begin{array}{l}\text { darunter mit } \\
\text { der Sprache: }\end{array}$ & & & & & & & \begin{tabular}{|l|}
$\begin{array}{l}\text { Teaching } \\
\text { language }\end{array}$ \\
\end{tabular} \\
\hline Tschechisch & 45636 & 41795 & 41603 & 41982 & 41621 & 42220 & Czech \\
\hline $\begin{array}{l}\text { Tschechisch } \\
\text { u. Deutsch } \\
\text { (bilingual) }\end{array}$ & 5 & 5 & 5 & 5 & 6 & - & $\begin{array}{l}\text { Czech and } \\
\text { German } \\
\text { (bilingual) }\end{array}$ \\
\hline $\begin{array}{l}\text { Tschechisch } \\
\text { u. Englisch } \\
\text { (bilingual) }\end{array}$ & & 29 & - & - & - & - & $\begin{array}{l}\text { Czech and } \\
\text { English } \\
\text { (bilingual) }\end{array}$ \\
\hline Polnisch & 128 & 112 & 112 & 118 & 112 & 114 & Polish \\
\hline Schüler insgesamt & 916575 & 794459 & 789486 & 794642 & 807950 & 827654 & Pupils, total \\
\hline $\begin{array}{l}\text { darunter mit } \\
\text { der Sprache: }\end{array}$ & & & & & & & \begin{tabular}{|l|} 
Teaching \\
language
\end{tabular} \\
\hline Tschechisch & 914596 & 792041 & 787749 & 792901 & 806159 & 825975 & Czech \\
\hline $\begin{array}{l}\text { Tschechisch } \\
\text { u. Deutsch } \\
\text { (bilingual) }\end{array}$ & 104 & 124 & 126 & 121 & 142 & - & $\begin{array}{l}\text { Czech and } \\
\text { German } \\
\text { (bilingual) }\end{array}$ \\
\hline $\begin{array}{l}\text { Tschechisch } \\
\text { u. Englisch } \\
\text { (bilingual) }\end{array}$ & . & 674 & - & - & - & - & $\begin{array}{l}\text { Czech and } \\
\text { English } \\
\text { (bilingual) }\end{array}$ \\
\hline Polnisch & 1875 & 1620 & 1611 & 1620 & 1649 & 1679 & Polish \\
\hline
\end{tabular}

Tabelle Nr. 4: Grundschulen nach der unterrichteten Sprache. (Quelle: Statistická ročenka České republiky 2014, Čsú) ${ }^{1}$

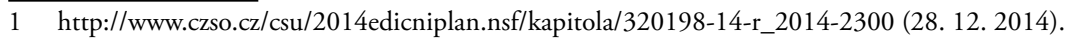


Interessant ist jedoch, dass sich die vom Tschechischen Amt für Statistik angeführten Angaben von denen von uns festgesellten grundsätzlich unterscheiden. Dem Statistischen Jahrbuch des Schulwesens (zum Herunterladen auf den WebSeiten des Tschechischen Schulministeriums) nach haben im Schuljahr 2010/2011 insgesamt 348439 Schüler Deutsch als Fremdsprache gelernt, und zwar 113849 in den Grundschulen, 224396 in den Mittelschulen und alle übrigen in anderen Schultypen (Musikschulen und Fachoberschulen). Die Statistik berücksichtigt erneut nicht, ob es sich um einsprachige oder zweisprachige (bilinguale) Schulen und SchülerInnen handelt. Angaben dieser Art werden bisher nicht erhoben und aktuellere Angaben waren leider nicht zu finden.

\begin{tabular}{|c|c|c|c|c|c|c|c|c|}
\hline & & \multicolumn{5}{|c|}{ Zahl der Schüler, die angegebene Sprache erlernen } \\
\hline Schultyp & $\begin{array}{c}\text { Schüler } \\
\text { insgesamt }^{2}\end{array}$ & Englisch & Franz. & Deutsch & Rus. & Latein & Span. & Ital. \\
\hline Insgesamt & $\mathbf{1} 156 \mathbf{4 3 4}$ & $\begin{array}{c}\mathbf{1 0 7 9} \\
\mathbf{3 2 4}\end{array}$ & $\mathbf{4 6} \mathbf{6 1 2}$ & $\mathbf{3 4 8} \mathbf{4 3 9}$ & $\mathbf{5 5 3 7 9}$ & $\mathbf{1 2} 134$ & $\mathbf{2 7} 751$ & $\mathbf{1 2 7 7}$ \\
\hline Grundschulen & 652404 & 628678 & 7428 & $\mathbf{1 1 3} \mathbf{8 4 9}$ & 24955 & - & 2316 & 182 \\
\hline Mittelschulen & 480159 & 430788 & 38438 & $\mathbf{2 2 4 3 9 6}$ & 29181 & 10231 & 24638 & 792 \\
\hline Konservatorien & 2929 & 2596 & 218 & 525 & - & 58 & 91 & 240 \\
\hline Fachobersch. & 20942 & 17262 & 528 & 9669 & 1243 & 1845 & 706 & 63 \\
\hline
\end{tabular}

Tabelle Nr. 5: Schüler, die Fremdsprachen erlernen - dem Schultyp nach, Stand zum 30. 9. 2010

Wie bereits erwähnt, dienen die von uns hier präsentierten Angaben nur dazu, dem Leser eine grobe Übersicht über bilinguale Schulen und Klassen sowie über die in der Tschechischen Republik unterrichteten Sprachen zu verschaffen. Für die Zwecke unserer Forschung hatten diese Daten leider keine Relevanz.

\subsection{Design der Forschung}

Nachdem wir uns mit der Fachliteratur zum Thema qualitative und quantitative Forschung (Chrástka 2007; Hendl 2012) bekannt gemacht hatten, erstellten wir einen Fragebogen, den wir für unsere Zwecke als die geeignetste Forschungsmethode betrachteten. Diese Methode ist sowohl für Forscher als auch für Probanden nicht besonders zeitaufwendig. Man kann rasch relativ große Datenmengen erhalten und meistens ist auch ein kompletter Rücklauf der Fragebögen gewährleistet. Dies war jedoch bei unserer Pilotstudie leider nicht der Fall.

Der von uns erstellte Fragebogen enthielt gezielte Fragen zur Problematik der Zweisprachigkeit und des Spracherwerbs, die an von uns ausgewählte Kinder oder

1 Jeder Schüler kann mehrmals gezählt werden, je nachdem, wie viele Sprachen er lernt.

2 Jeder Schüler wird nur einmal gezählt, ohne Hinsicht darauf, wie viele Sprachen er lernt. 
SchülerInnen gerichtet waren. Es handelte sich um Fragen, mit deren Hilfe wir Antworten auf die zentralen Fragen unseres Forschungsvorhabens ermitteln wollten. Konkret ging es uns darum festzustellen, wie unsere Probanden die jeweilige(n) Sprache(n) erworben haben, ob sie monolingual oder bilingual (multilingual) sind, wie zu Hause gesprochen wird etc. Diese Fragebögen schickten wir anschließend an die ausgewählten Kindergärten, Kitas und Schulen, mit der Bitte, im Falle von Kindern in Kindergärten und Kitas die Fragebögen von Kindergärtnerinnen bzw. den Eltern ausfüllen zu lassen, jedoch auf Grund der von den Kindern erhaltenen Antworten. Für die Hauptschulen und in Mittelschulen haben wir vorausgesetzt, dass die SchülerInnen im Stande sind, die zugeschickten Fragebögen selbst auszufüllen. Im nächsten Schritt haben uns die Kollegen aus den jeweiligen Schulen die ausgefüllten Fragebögen zurückgeschickt, worauf wir mit unserer Analyse begonnen haben.

\subsection{Analyse der Daten}

Von den Daten ausgehend, die uns vom Tschechischen Landesamt für Statistik und vom Tschechischen Schulministerium zur Verfügung gestellt wurden, haben wir uns in unserer Pilotstudie zum Ziel gesetzt, die aktuelle Lage der Unterstützung des tschechisch-deutschen Bilingualismus zu betrachten. Wie bereits mehrmals gesagt, stellten wir nur die Institutionen in den Fokus, die zur Bewahrung und zur Unterstützung des tschechisch-deutschen Bilingualismus beitragen. Es ging dabei um Programme der präprimären, primären und sekundären Bildung. Die tertiäre Bildung haben wir zunächst außen vor gelassen.

Das typische Merkmal eines bilingualen Bildungsganges ist der sog. bilinguale Sachunterricht. Darunter verstehen wir einen zweisprachig realisierten Sachunterricht in einem bestimmten Fach, normalerweise in Erdkunde, Geschichte, Biologie oder ähnlichen Fächern. Bei solch einem Unterricht werden zwei Sprachen parallel benutzt: die Muttersprache der Schüler und die Fremdsprache. Mit steigender Jahrgangsstufe nimmt der Anteil der Muttersprache immer mehr ab und schließlich wird nur noch mit Hilfe der Fremdsprache kommuniziert. Dabei sind zwei Formen zu unterscheiden: eine, bei der sich ein qualifizierter muttersprachlicher und ein qualifizierter fremdsprachiger Lehrer im Unterricht abwechseln, und eine weitere, bei der nur ein qualifizierter fremdsprachiger Lehrer den Unterricht leitet. Solch ein Unterricht stellt jedoch außergewöhnlich hohe Ansprüche an die Lehrkraft, die nicht nur das Fachwissen vermitteln, sondern auch in der Zielsprache sehr kompetent sein muss.

Bei unserer Forschung hat uns interessiert, wie viele Schüler insgesamt in der jeweiligen Ausbildungsstufe in einem bilingualen tschechisch-deutschen Ausbildungsprogramm ausgebildet werden, wie viele davon entweder ausschließlich aus tschechischen Familien stammen (und mit Deutsch erst im Rahmen einer 
institutionellen Ausbildung begonnen haben und damit als sukzessiv bilingual gelten können) und wie viele aus rein deutschen Familien bzw. aus gemischten (tschechisch-deutschen/österreichischen/schweizerischen) Familien stammen (und deshalb simultan zweisprachig aufgewachsen sind). In diesem Punkt sind wir von der Annahme ausgegangen, dass Kinder/Schüler aus bilingualen (im idealen Fall aus tschechisch-deutschen Familien) in den von uns untersuchten Schulen dominieren werden. Diese Annahme sollte durch die Untersuchung überprüft werden.

Gleichzeitig hat uns auch interessiert, ob die Zahlen der Deutsch lernenden Kinder/Schüler, wie wir sie selbst ermittelt haben, mit den Zahlen übereinstimmen, wie sie im Statistischen Jahrbuch des Schulwesens im Jahr 2010 angegeben werden.

Insgesamt haben wir 19 Kindergärten/Kitas, 5 Hauptschulen und 5 Gymnasien mit einem bilingualen, deutschfördernden Ausbildungsprogramm angesprochen. Antworten auf unsere Fragen haben wir jedoch nur von 6 Kindergärten/Kitas, einer Hauptschule und zwei Gymnasien erhalten. Bei den Kindergärten/Kitas handelte es sich sowohl um öffentliche als auch um private Einrichtungen, die zur Förderung der deutschen Sprache von früher Kindheit an beitragen. Alle Kindergärten/Kitas waren ausschließlich tschechische Einrichtungen im Sinne des tschechischen Rechts, keine davon hatte einen ausländischen Stifter. In zwei Fällen handelte es sich um Kindergärten aus dem Grenzgebiet.

Folgende Graphiken stellen die Gesamtzahl der Kinder in den einzelnen Kindergärten/Kitas, die Zahl der Kinder aus einsprachigen tschechischen Familien und die Zahl der Kinder aus zweisprachig tschechisch-deutschem/tschechischösterreichischem Umfeld dar, wo jeder Elternteil den Träger eines der Codes repräsentiert.

\section{Kindergarten 1 - 32 Kinder insgesamt}

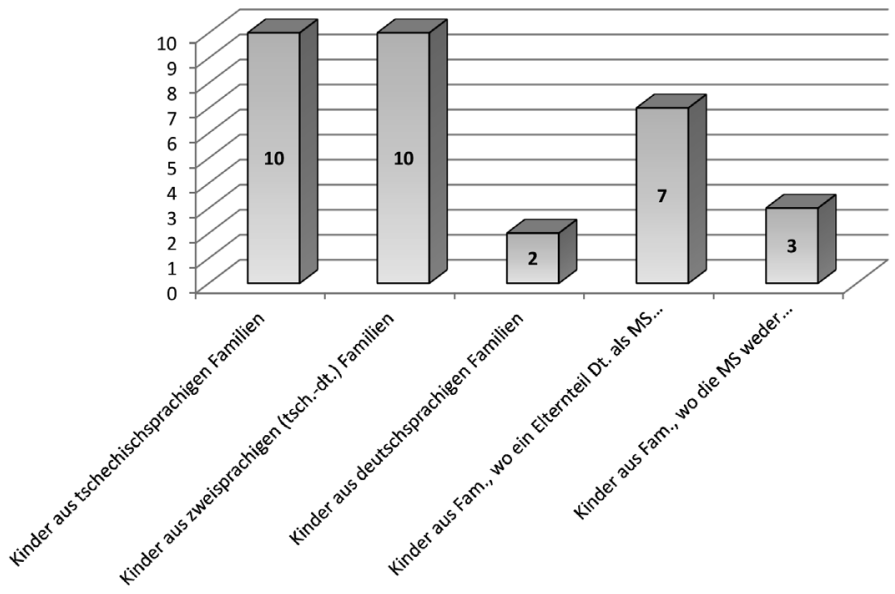

Graphik Nr. 1: Kindergarten Nr. 1- Region Prag 


\section{Kindergarten 2 - 18 Kinder insgesamt}

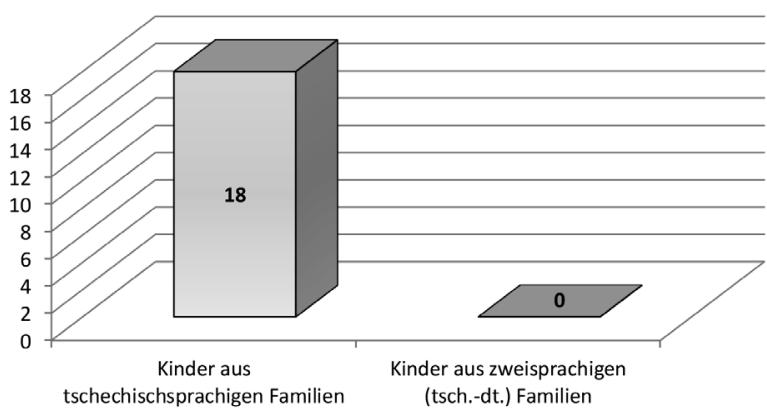

Graphik Nr. 2: Kindergarten Nr. 2 - Region Hochland

\section{Kindergarten 3 - 14 Kinder insgesamt}

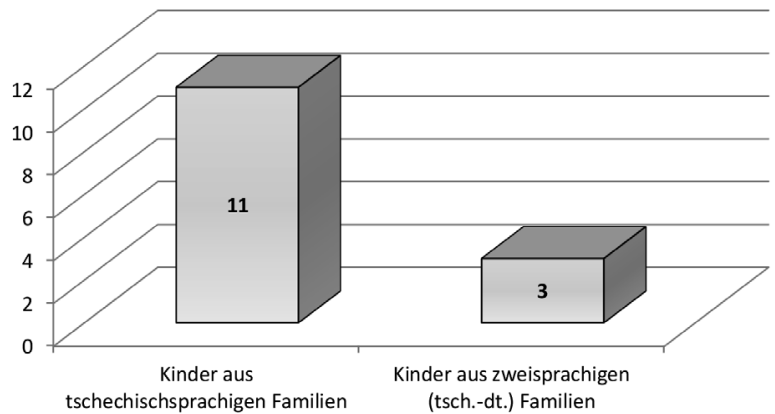

Graphik Nr. 3: Kindergarten Nr. 3 - Region Prag

\section{Kindergarten 4 - 24 Kinder insgesamt}

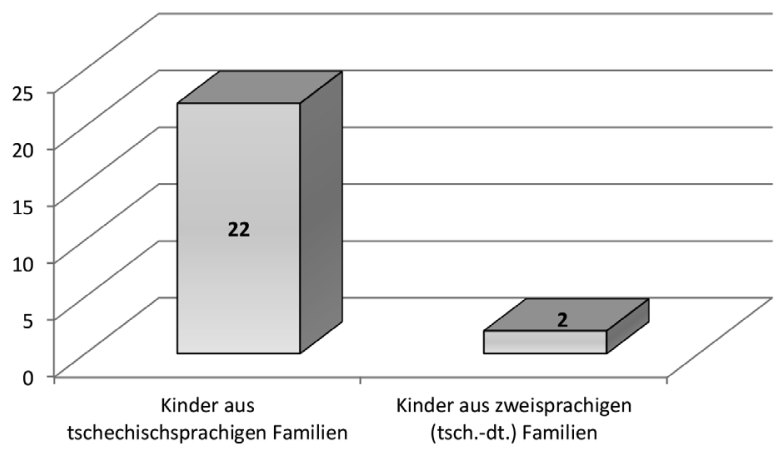

Graphik Nr. 4: Kindergarten Nr. 4 - Region Reichenberg 


\section{Kindergarten 5 - 22 Kinder insgesamt}

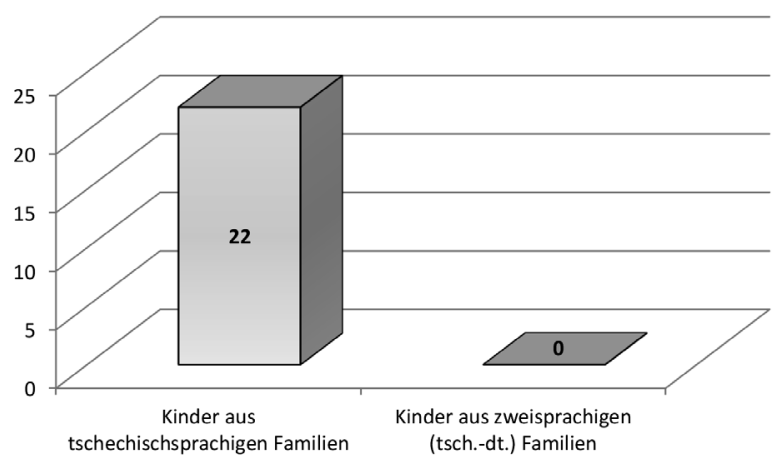

Graphik Nr. 5: Kindergarten Nr. 5 - Region Südböhmen

Kindergarten 6 - 28 Kinder insgesamt

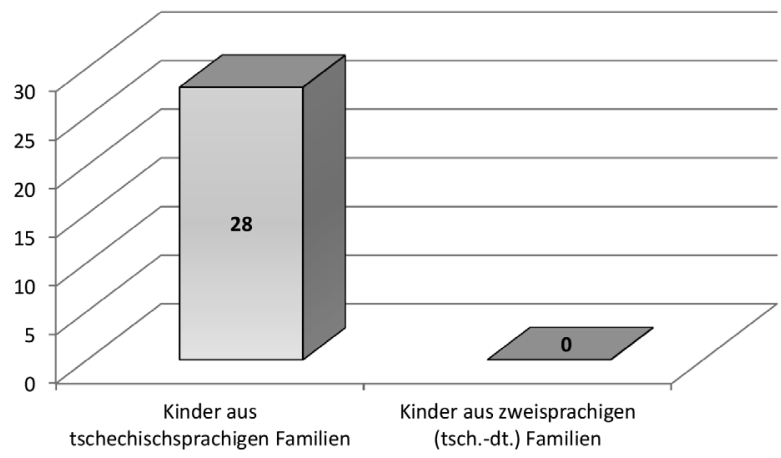

Graphik Nr. 6: Kindergarten Nr. 6-Region Reichenberg

Wie wir bereits oben angeführt haben, haben wir von allen angesprochenen Hauptschulen nur zwei Reaktionen zurückerhalten. Es handelte sich um eine Hauptschule aus der Region Südböhmen und eine aus Prag. Eine davon war öffentlich-rechtlich, die andere privat, jedoch aus tschechischen finanziellen Mitteln unterstützt. 


\section{Hauptschule 1 - 32 Schüler insgesamt}

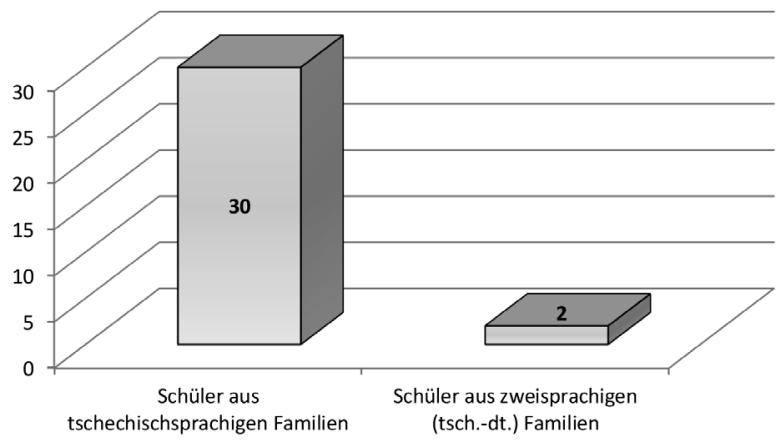

Graphik Nr. 7: Hauptschule Nr. 1 - Region Südböhmen

\section{Hauptschule 2 - 198 Schüler insgesamt}

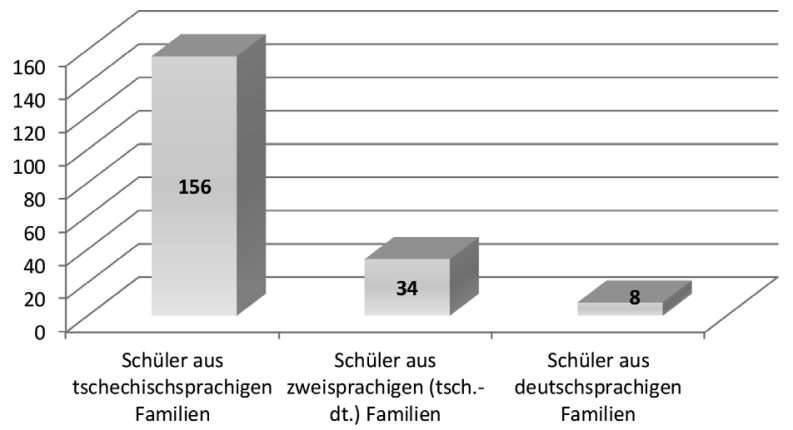

Graphik Nr. 8: Hauptschule Nr. 2 - Region Prag

Unter den Gymnasien, die wir angesprochen haben und die uns relevante Daten zur Verfügung gestellt haben, befand sich sowohl ein Gymnasium, das mit der Bundesrepublik Deutschland zusammenarbeitet, als auch eines, das mit der Republik Österreich kooperiert. Im Prinzip handelte es sich um sechsjährige private Gymnasien mit zwei Unterrichtssprachen, Tschechisch und Deutsch, wobei der Unterricht ausgewählter Fächer und das Abitur dann in Deutsch realisiert werden. 


\section{Gymnasium 1 - 148 Schüler insgesamt}

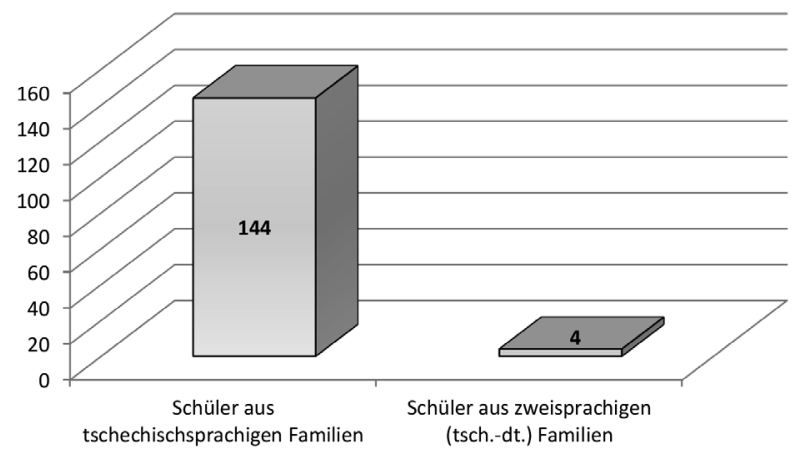

Graphik Nr. 9: Gymnasium Nr. 1 - Region Prag

\section{Gymnasium 2 - 139 Schüler insgesamt}

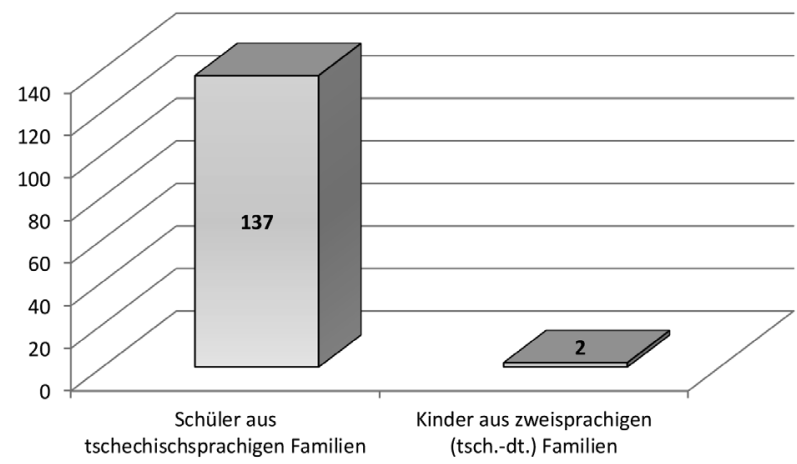

Graphik Nr. 10: Gymnasium Nr. 2 - Region Südmähren

\subsection{Zusammenfassende Auswertung der Pilotstudie}

Auf der Grundlage der von uns ausgewählten Gruppe von Befragten sind wir hinsichtlich des simultanen bzw. sukzessiven Bilingualismus $\mathrm{zu}$ folgenden Ergebnissen gekommen:

Aus der Gesamtzahl der 138 Kinder, die die untersuchten Kindergärten/Kitas besuchten, stammten 80,4 \% der Kinder aus rein tschechischen Familien, fast $11 \%$ der Kinder aus einem zweisprachigen (tschechisch-deutschen) sozialen Umfeld und nur ungefähr 1,5\% aus einer rein deutschsprachigen Familie. Die restlichen Kinder stammten aus Familien, von denen ein Elternteil Deutsch als Muttersprache 
spricht, der andere eine andere Fremdsprache, oder aus Familien, bei denen die Muttersprache weder Tschechisch noch Deutsch ist.

\section{Kinder in Kindergärten/Kitas - insgesamt 138}

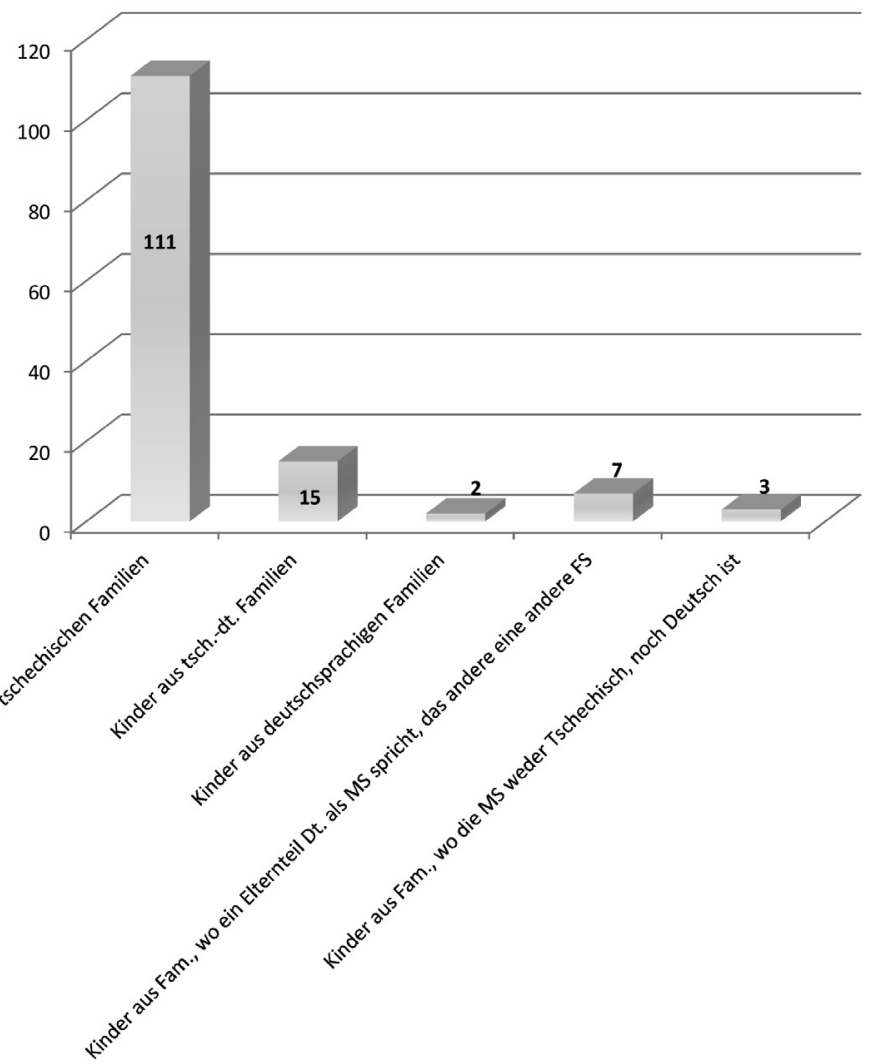

Graphik Nr. 11: Auftreten des tsch.-dt. Bilingualismus im Vorschulalter (Legende: Dt. Deutsch, MS - Muttersprache, FS - Fremdsprache)

Die Angaben, die wir von den Hauptschulen erhalten haben, haben leider wegen der geringen Anzahl der Befragten nur eine begrenzte Aussagekraft. Es gelang uns festzustellen, dass im Fall dieser zwei Schulen fast $81 \%$ der Schüler aus rein tschechischen Familien stammen, 15,6\% tschechisch-deutsch zweisprachig aufwachsen und ca. 3,5\% aus einer rein deutschsprachigen Familie kommen. 


\section{Schüler in Haupschulen - 230 insgesamt}

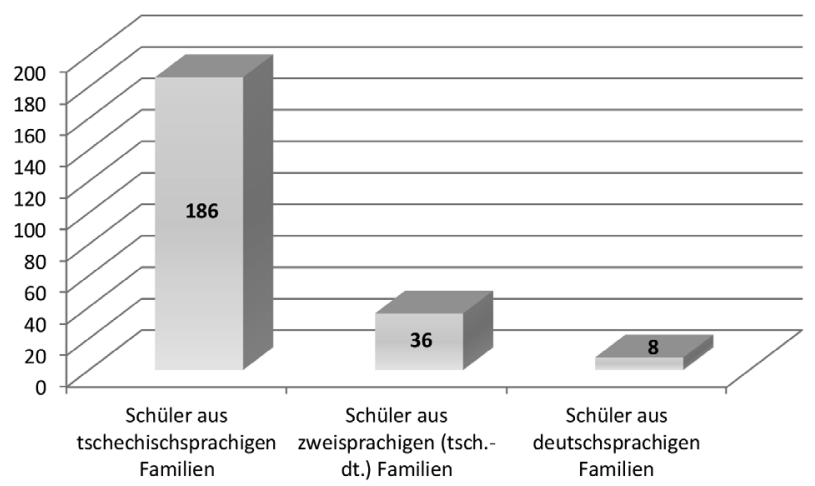

Graphik Nr. 12: Auftreten des tschechisch-deutschen Bilingualismus im Hauptschulalter

Etwas umfangreichere Angaben haben wir von den zweisprachig orientierten Gymnasien erhalten. Es muss aber hinzugefügt werden, dass wir uns auch hier eine intensivere und aussagekräftigere Zusammenarbeit erwartet hätten. Aus der Gesamtzahl von 287 Schülern, die in den zweisprachigen Bildungsprogrammen beschult werden, waren nur 2,09\% simultan zweisprachig (tschechisch-deutsch).

\section{Schüler in Gymnasien - 287 insgesamt}

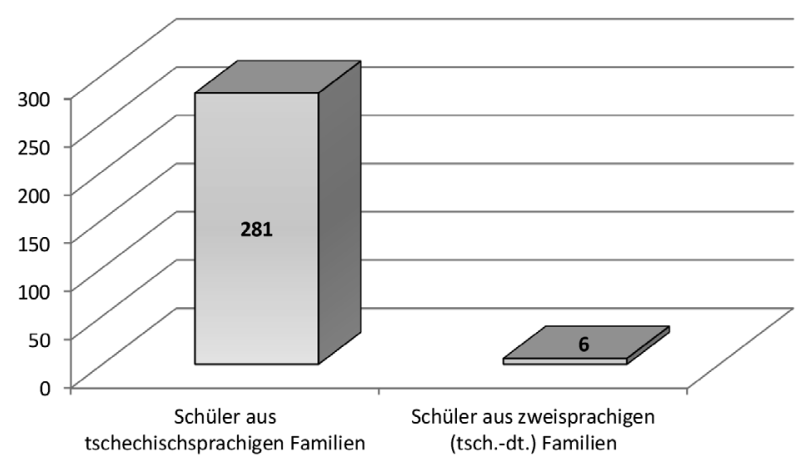

Graphik Nr. 13: Auftreten des tschechisch-deutschen Bilingualismus im Gymnasialalter

Aus der von uns durchgeführten Studie geht hervor, dass unsere dritte Annahme (dass die meisten Schüler in bilingualen Bildungsprogrammen simultan bilinguale Kinder sind) gewissermaßen illusorisch war: Das Gros der Schüler der von uns untersuchten Institutionen stellen sukzessiv bilinguale Sprecher dar, die ihre 
fremdsprachliche Kompetenz erst im Rahmen der institutionellen Ausbildung erwerben, nicht simultan bilingualen Sprecher, wie wir ursprünglich erwartet hatten. Auch die beiden anderen Annahmen, dass nämlich die angesprochenen Schulen von ausländischen Subjekten finanziell unterstützt werden und dass das Schulministerium genaue Daten über zweisprachige Bildungsprogramme und über Schüler, die im Rahmen von diesen Programmen ausgebildet werden, sammelt, erwiesen sich als falsch.

\section{Resümee}

Aus der angeführten Analyse der Daten, die uns das Tschechische Landesamt für Statistik zur Verfügung gestellt hat, können wir in den letzten Jahren einen eindeutigen Rückgang sowohl bei den in Tschechien lebenden und sich zur deutschen Nationalität bekennenden Einwohnern als auch einen Rückgang von Einwohnern, die Deutsch auf dem Gebiet der Tschechischen Republik als Muttersprache sprechen, beobachten.

Zum Zwecke der Wiederbelebung des tschechisch-deutschen Bilingualismus, der bei uns eine lange Tradition hat und mit unserem Land eng verbunden ist, entstand eine ganze Reihe von Institutionen, die sich auf eine zweisprachige tschechisch-deutsche Erziehung konzentrieren. Zu diesen gehören Kindergärten (Kitas), Hauptschulen, Mittelschulen, aber auch einige Hochschulen.

Vor Beginn unserer Pilotstudie hatten wir angenommen, dass die Schüler der von uns befragten Institutionen vor allem simultan zweisprachige Sprecher sein werden, d. h. Schüler, die parallel beide Sprachcodes benutzen (in unserem Fall Tschechisch und Deutsch), und zwar beide als Muttersprachen. Die Ergebnisse, die sich aus unserer Pilotstudie herauskristallisieren, zeigen, dass diese Annahme falsch ist. Dabei sind wir uns selbstverständlich dessen bewusst, dass es sich im Fall des tschechisch-deutschen Bilingualismus um ein sehr kompliziertes Phänomen handelt, das eine ausführlichere und präzisere Analyse verdienen würde. Das Ziel unserer Pilotstudie war lediglich, einen Einstieg in die Problematik bereitzustellen. Diese erste Ausarbeitung soll zu einer detaillierten Betrachtung und zu einem intensiveren Studium dieses Themenbereiches anregen.

\section{Literaturverzeichnis}

Apeltauer, Ernst (1987): Gesteuerter Zweitspracherwerb: Voraussetzungen und Konsequenzen für den Unterricht. München, Hueber Verlag.

Bialystok, Ellen (2001): Bilingualism in Development. Language, Literacy and Cognition. Cambridge, Cambridge University Press.

Černý, Jiří (1996): Dějiny lingvistiky. Olomouc, Votobia.

Europarat/Rat für kulturelle Zusammenarbeit (2001): Gemeinsamer europäischer Referenzrahmen für Sprachen: lernen, lehren, beurteilen. Berlin, Langenscheidt. 
Harding-Esch, Edith / Riley, Philip (2008): Bilingvni rodina. Praha, Portál.

Hendl, Jan (2012): Kvalitativni výzkum: základni teorie, metody a aplikace. 3. Aufl. Praha, Portál.

Chráska, Miroslav (2007): Metody pedagogického výzkumu: základy kvantitativního výzkumu. Praha, Grada.

Jelínek, Stanislav (2004-2005; 2005-2006): K některým otázkám bilingvismu I. a II. In: Cizí jazyky 48/5/2004-2005. S. 153-155 und 49/1/2005-2006. S. 8-11.

Jonekeit, Sylvie / Kielhöfer, Bernd (2002): Zweisprachige Kindererziehung. Tübingen, Stauffenburg.

Lachout, Martin (2014): Cesta od nehomogennosti jazykového systému cizího jazyka k jeho potenciálni homogennosti. Praha, Metropolitan University Prague Press.

McNamara, John (1967): The bilingual's linguistic performance. A psychological overview. Journal of Social 23/1967. S. 58-77.

Muhić, Jasmin (2004): Interkulturni poradenstvi 2004: Projekt MŠMT. Praha, Institut pedagogicko-psychologického poradenství ČR.

Průcha, Jan / Walterová, Eliška / Mareš, Jiří (2003): Pedagogický slovník. Praha, Portál.

Skutnabb-Kangas, Tove (1984): Bilingualism or Not - The Education of Minorities. Clevedon/ UK, Multilingual Matters.

Sternberg, Robert J. (2009): Kognitivni psychologie. Praha, Portál.

Štefánik, Jozef (2000): Jeden človek, dva jazyky - Dvojjazyčnost'u detí-predsudky a skutočnost'. Bratislava, AEP.

\section{Tabellen und Graphiken:}

Quelle: ČSÚ, heruntergeladen am 28.05.2015, www.czso.cz www.msmt.cz

\author{
Martin Lachout \\ Katedra cizích jazyků \\ Metropolitní univerzita Praha \\ Dubečská 900/10 \\ 10031 Praha 10 \\ martin.lachout@mup.cz
}

Katedra germanistiky
FF UJEP
Pasteurova 13
40096 Ústí nad Labem
martin.lachout@ujep.cz 